\title{
LATTICE AUTOMORPHISMS OF SEMI-SIMPLE LIE ALGEBRAS
}

\author{
Dedicated to the memory of Hanna Neumann \\ D. W. BARNES
}

(Received 12 April 1972)

Communicated by M. F. Newman

\section{Introduction}

Let $L$ be a Lie algebra over the field $F$. A lattice automorphism of $L$ is an automorphism $\phi: \mathscr{L}(L) \rightarrow \mathscr{L}(L)$ of the lattice $\mathscr{L}(L)$ of all subalgebras of $L$. We seek to describe the lattice automorphisms in terms of maps $\sigma: L \rightarrow L$ of the underlying algebra. A semi-automorphism $\sigma$ of $L$ is an automorphism of the algebraic system consisting of the pair $(F, L)$, that is, a pair of maps $\sigma: F \rightarrow F$, $\sigma: L \rightarrow L$ preserving the operations. Thus $\sigma: F \rightarrow F$ is an automorphism of $F$ and $(x+y)^{\sigma}=x^{\sigma}+y^{\sigma},(x y)^{\sigma}=x^{\sigma} y^{\sigma},(\lambda x)^{\sigma}=\lambda^{\sigma} x^{\sigma}$ for all $x, y \in L$ and $\lambda \in F$. Clearly, any semi-automorphism of $L$ induces a lattice automorphism. To study a given lattice automorphism $\phi$, we select a semi-automorphism $\sigma$ such that $\phi \sigma^{-1}$ fixes certain subalgebras, and so we reduce the problem to the investigation of lattice automorphisms leaving these subalgebras fixed.

Except where otherwise stated, we suppose that $F$ is an algebraically closed field of characteristic 0 and that $L$ is semi-sin ple of rank $r \geqq 3$. For $x \in L$, we denote by $x_{s}, x_{n}$ the semi-simple and nil parts ${ }^{1}$ respectively of $x$. We shall prove

THEOREM. Let $L$ be a semi-simple Lie algebra of rank $\geqq 3$ over an algebraically closed field $F$ of characteristic 0 . For $t \in F, t \neq 0$, let $\tau(t): L \rightarrow L$ be the map defined by $x^{\tau(t)}=x_{s}+t x_{n}$ for $x \in L$. Let $\phi$ be a lattice automorphism of $L$. Then there exists a unique semi-automorphism $\sigma$ of $L$ and a unique $t \in F, t \neq 0$ such that $\sigma \tau(t)$ induces $\phi$. Conversely, for all semi-automorphisms $\sigma$ and all $t \in F, t \neq 0$, the map $\sigma \tau(t)$ induces a lattice automorphism of $L$.

If $A$ is a subset of $L$, we denote by $\langle A\rangle$ the subspace spanned by $A$. We denote by $\langle\langle A\rangle\rangle$ the subalgebra generated by $A$. We say that the lattice automorphism $\phi$ fixes the subalgebra $A$ if $A^{\phi}=A$. Let $A$ be any sul space of $L$. We say that $\phi$ is the identity on $A$ if $U^{\varphi}=U$ for all subalgebras $U$ of $L$ contained in $A$, that is, if $\langle a\rangle^{\phi}=\langle a\rangle$ for all $a \in A$. We say that $\phi$ is linear (semi-linear) on $A$ if

1. See Jacobson [3] p. 98. 
there exists a linear (respectively semi-linear) transformation $\sigma: A \rightarrow A$ such that $\langle a\rangle^{\phi}=\left\langle a^{\sigma}\right\rangle$ for all $a \in A$.

We note that, if $A$ is a subalgebra fixed by $\phi, \operatorname{dim} A \geqq 3$, and if every subspace of $A$ is a subalgebra, then by the Fundamental Theorem of Projective Geometry, $\phi$ is semi-linear on $A$. If $\phi$ is semi-linear on $A$ and there exists a 2-dimensional subspace $B \subseteq A$ on which $\phi$ is linear, then $\phi$ is linear on $A$.

\section{Reduction}

Let $H$ be a Cartan subalgebra of $L$. We denote by $h_{\alpha}$ the scaled star vector defined by

$$
\left(h, h_{\alpha}\right)=\alpha(h) /(\alpha, \alpha)
$$

for all $h \in H$. This departure from the usual notation is convenient for our purposes. For any choice of root vector $e_{\alpha}$, we have $h_{\alpha} e_{\alpha}=e_{\alpha}$. We now choose root vectors $e_{\alpha}$ such that $e_{-\alpha} e_{\alpha}=h_{\alpha}$ and such that all the multiplication constants $v_{\alpha, \beta}$ (defined when $\alpha, \beta, \alpha+\beta$ are roots by $e_{\alpha} e_{\beta}=v_{\alpha, \beta} e_{\alpha+\beta}$ ) are rational. We choose an order relation $<$ on the roots in the usual way. We put $K_{\alpha}=\operatorname{ker} \alpha$.

Definition 2.1. We say that the lattice automorphism $\phi$ is reduced with respect to $H,\left\{e_{\alpha} \mid \alpha \operatorname{root}\right\},<$ if $\phi$ is the identity on $H$ and fixes $\left\langle h_{\alpha}+e_{\alpha}\right\rangle$ for every fundamental root $\alpha$.

In $\S 4$, we shall show that the concept of a reduced lattice automorphism is in fact independent of the choices of $H,\left\{e_{\alpha} \mid \alpha\right.$ root $\}$ and of $<$. If $\left\langle e_{\gamma}\right\rangle$ is a root space for $H$, then $\left\langle e_{\gamma}\right\rangle^{\phi}$ is a root space for $H^{\phi}$ by Barnes [1], Lemma 4. If $\phi$ is reduced, we have that $\phi$ fixes $\left\langle h_{\alpha}\right\rangle,\left\langle h_{\alpha}+e_{\alpha}\right\rangle$ and so also $\left\langle e_{\alpha}\right\rangle$ for each fundamental root $\alpha$. Since $\phi$ also preserves sums of roots ([1], proof of Theorem 2), it follows that $\phi$ fixes $\left\langle e_{\gamma}\right\rangle$ for all roots $\gamma$.

Lemma 2.2. Let $\phi$ be a lattice automorphism of L. Then there exists a unique semi-automorphism $\sigma$ of $L$ such that $\phi^{\sigma-1}$ is reduced with respect to given $H,\left\{e_{\alpha}\right\},<$.

Proof. $H^{\phi}$ is a Cartan subalgebra, $\left\langle e_{\alpha}\right\rangle^{\phi}$ is the root space of a root $\alpha^{\phi}$ for $H^{\phi}$, and the correspondence $\alpha \rightarrow \alpha^{\phi}$ preserves sums. Therefore there exists an automorphism $\sigma_{1}$ of $L$ such that $H^{\sigma^{\prime}}=H^{\phi}$ and $\left\langle e_{\alpha}^{\sigma^{\prime}}\right\rangle=\left\langle e_{\alpha}\right\rangle^{\phi}$ for all roots $\alpha$. Thus $\phi \sigma_{1}^{-1}$ fixes $H$ and all the root spaces $\left\langle e_{\alpha}\right\rangle$. This implies that $\phi \sigma_{1}^{-1}$ fixes $\left\langle h_{\alpha}\right\rangle$ for all $\alpha$. Since $H$ is abelian and $\operatorname{dim} H \geqq 3, \phi \sigma_{1}^{-1}$ is semi-linear on $H$. Let $\alpha_{1}, \cdots, \alpha_{r}$ be the fundamental roots and put $h_{i}=h_{\alpha_{i}}$. The semi-linear transformation fixes all the $\left\langle h_{i}\right\rangle$, and so has the form

$$
\sum_{i=1}^{r} x_{i} h_{i} \rightarrow \sum_{i=1}^{r} \lambda_{i} x_{i}^{\tau} h_{i}
$$

where $\tau$ is an automorphism of $F$ and $\lambda_{1}, \cdots, \lambda_{r} \in F$. We now prove $\lambda_{i}=\lambda_{j}$ for all $i, j$. 
Suppose $\alpha_{i}+\alpha_{j}=\beta$ is a root. Then $h_{\beta}=x h_{\alpha_{i}}+y h_{\alpha_{j}}$, where $x=\left(\alpha_{i}, \alpha_{i}\right) /(\beta, \beta)$ and $y=\left(\alpha_{j}, \alpha_{j}\right) /(\beta, \beta)$. Since $x, y$ are rational, $x^{\tau}=x, y^{\tau}=y$ and the semi-linear transformation sends $h_{\beta}$ to $\lambda_{i} x h_{\alpha_{i}}+\lambda_{j} y h_{\alpha_{j}}$. But $\left\langle h_{\beta}\right\rangle$ is fixed by $\phi \sigma_{1}^{-1}$ and therefore $\lambda_{i}=\lambda_{j}$.

Suppose $\alpha_{i}+\alpha_{j}$ is not a root. Then $e_{\alpha_{i}} e_{\alpha_{j}}=0,\left\langle e_{\alpha_{i}}, e_{\alpha_{j}}\right\rangle$ is a subalgebra fixed by $\phi \sigma_{1}^{-1}$ and it follows that $\phi \sigma_{1}^{-1}$ permutes the subalgebras $\left\langle e_{\alpha_{i}}+x e_{\alpha_{1}}\right\rangle$ for $x \neq 0$. The subalgebra $\left\langle h_{i}+y h_{j}\right\rangle$ generates with $\left\langle e_{\alpha_{i}}+x e_{\alpha_{j}}\right\rangle$ a 2-dimensional subalgebra ff and only if $y=1$. Since this property is preserved by $\phi \sigma_{1}^{-1},\left\langle h_{i}+h_{j}\right\rangle$ is fixed by $\phi \sigma_{1}^{-1}$ and we have $\lambda_{i}=\lambda_{j}$.

We have now shown that $\phi \sigma_{1}^{-1}$ is given on $H$ by the semi-linear map

$$
\sum_{i=1}^{r} x_{i} h_{i} \rightarrow \sum_{i=1}^{r} x_{i}^{\tau} h_{i}
$$

We define $\sigma_{2}: L \rightarrow L$ by

$$
\left(\sum_{i=1}^{r} x_{i} h_{i}+\sum_{\gamma \text { root }} y_{\gamma} e_{\gamma}\right)^{\sigma_{2}}=\sum_{i=1}^{r} x_{i}^{\tau} h_{i}+\sum_{\gamma \text { root }} y_{\gamma}^{\tau} e_{\gamma}
$$

Since the multiplication constants are rational, they are fixed uncer $\tau$ and $\sigma_{2}$ is a semi-automorphism. We now have that $\phi \sigma_{1}^{-1} \sigma_{2}^{-1}$ is the identity on $H$ and fixes all the $\left\langle e_{\gamma}\right\rangle$.

Let $\lambda_{1}, \cdots, \lambda_{r}$ be non-zero elements of $F$. For each root

$$
\gamma=\sum_{i=1}^{r} m_{i} x_{i},\left(m_{i} \in \mathbb{Z},\right) \text { put } \lambda_{\gamma}=\prod_{i=1}^{r} \lambda_{i}^{m_{i}} .
$$

Then the map $\sigma_{3}: L \rightarrow L$ defined by

$$
\left(h+\sum_{\gamma} x_{\gamma} e_{\gamma}\right)^{\sigma_{3}}=h+\sum_{\gamma} x_{\gamma} \lambda_{\gamma} e_{\gamma}
$$

for $h \in H, x_{y} \in F$, is an automorphism of $L$ and every automorphism of $L$ which is the identity on $H$ has this form. We have $\left\langle h_{i}+e_{\alpha_{i}}\right\rangle^{\varphi \sigma_{1}^{-1} \sigma_{2}-1}=\left\langle h_{i}+\mu_{i} e_{\alpha_{i}}\right\rangle$ for some $\mu_{i} \neq 0$. Put $\lambda_{i}=1 / \mu_{i}(i=1,2, \cdots, r)$ and then $\sigma=\sigma_{3} \sigma_{2} \sigma_{1}$ is a semi-automorphism with the required properties.

Suppose $\sigma^{\prime}$ is another such semi-automorphism. Then $\sigma^{\prime} \sigma^{-1}$ is a semiautomorphism of $L$. For all $h \in H$,

$$
\langle h\rangle^{\phi}=\langle h\rangle^{\sigma}=\langle h\rangle^{\sigma}
$$

and $\sigma^{\prime} \sigma^{-1}$ is a scalar multiple of the identity on $H$. As $\sigma^{\prime} \sigma^{-1}$ is linear on $H$, it is also linear on $L$ and so is an automorphism of $L$. Since we also have $\left\langle h_{i}+e_{\alpha_{1}}\right\rangle^{\sigma \sigma-1}$ $=\left\langle h_{i}+e_{\alpha_{i}}\right\rangle$, it follows that the permutation of the roots given by $\sigma^{\prime} \sigma^{-1}$ is the identity and hence, that $h^{\sigma \sigma-1}$ is the scaled star vector of the root $\alpha_{i}$, that is $h_{i}^{\sigma^{\prime} \sigma-1}=h_{i}$ and $\sigma^{\prime} \sigma^{-1}$ is the identity on $H$. Thus $\sigma^{\prime} \sigma^{-1}$ is an automorphism of the form $\left(^{*}\right)$ above, and, since $\left\langle h_{i}+e_{\alpha_{i}}\right\rangle^{\sigma / \sigma-1}=\left\langle h_{i}+e_{\alpha_{i}}\right\rangle$, it follows that $\sigma^{\prime} \sigma^{-1}=1$. 


\section{The algebra $\mathfrak{U}_{1}$}

Suppose the lattice automorphism $\phi$ of $L$ is reduced with respect to $H$. If $\alpha$ is a root, then $\left\langle h_{\alpha}, e_{\alpha}, e_{-\alpha}\right\rangle$ is a subalgebra of type $\mathfrak{U}_{1}$ and $\phi$ gives a lattice automorphism of $\left\langle h_{\alpha}, e_{\alpha}, e_{-\alpha}\right\rangle$. Consequently it is of interest to determine the lattice automorphisms of $\mathfrak{A}_{1}$.

Let $S$ be the Lie algebra $\langle h, e, f\rangle$ with defining relations $h e=e, h f=-f$, $e f=h$. We consider the projective plane whose points and lines are the 1 and 2-dimensional subspaces of $S$. The conic defined by the vanishing of the Killing form is the set of 1-dimensional subalgebras which are not Cartan subalgebras. The 2-dimensional subalgebras are the tangents to the conic, each point of the conic being a root space for the Cartan subalgebras on the tangent at the point.

Let $\phi$ be a lattice automorphism of $S$. Then $\phi$ permutes the points of the conic. Since each point not on the conic is the intersection of two tangents, this permutation determines $\phi$ completely. Since the tangents are the only 2-dimensional subalgebras, every permutation of the points of the conic gives a lattice automorphism of $S$.

LEMMA 3.1. Let $\phi$ be a latice automorphism of $S$ which fixes $\langle h\rangle$ and is linear on $\langle h, e\rangle$. Suppose $\langle h+e\rangle^{\phi}=\langle h+\lambda e\rangle$. Then $\phi$ is given by

$$
\langle x h+y e+z f\rangle^{\phi}=\left\langle x h+\lambda y e+\frac{1}{\lambda} z f\right\rangle
$$

for $x, y, z \in F$.

Proof. For $u=x h+y e+z f,(u, u)=2\left(x^{2}+2 y z\right)$. Thus the conic can be expressed parametrically

$$
x=\theta, y=-\frac{1}{2} \theta^{2}, z=1 .
$$

The tangent to the conic at $\left(\theta,-\frac{1}{2} \theta^{2}, 1\right)$ has equation $\theta x+y-\frac{1}{2} \theta^{2} z=0$ and meets $\langle h, e\rangle$ in $\langle h+\theta e\rangle$. But $\langle h+\theta e\rangle^{\phi}=\langle h+\lambda \theta e\rangle$. Thus $\left(\theta,-\frac{1}{2} \theta^{2}, 1\right)^{\phi}$ $=\left(\lambda \theta,-\frac{1}{2} \lambda^{2} \theta^{2}, 1\right)$. The tangents from $\left(x_{0}, y_{0}, z_{0}\right)$ meet the conic at its points of intersection with the polar line $x_{0} x+z_{0} y+y_{0} z=0$, that is at the points $\left(\theta,-\frac{1}{2} \theta^{2}, 1\right)$ for $\theta$ satisfying $x_{0} \theta-\frac{1}{2} z_{0} \theta^{2}+y_{0}=0$. The tangents from $\left(x_{1}, y_{1}, z_{1}\right)=\left(x_{0}, y_{0}, z_{0}\right)^{\phi}$ meet the conic in the points $\left(\theta^{\prime},-\frac{1}{2} \theta^{\prime 2}, 1\right)$ for $\theta^{\prime}$ satisfying $x_{0} \theta^{\prime} / \lambda+z_{0}$

Therefore

$$
x_{0} \frac{\theta^{\prime}}{\lambda}-\frac{1}{2} z_{0}\left(\frac{\theta^{\prime}}{\lambda}\right)^{2}+y_{0}=0 \text {. }
$$

and

$$
x_{1}: y_{1}: z_{1}=x_{0} / \lambda: y_{0}: z_{0} / \lambda^{2}
$$

$$
\left\langle x_{0} h+y_{0} e+z_{0} f\right\rangle^{\phi}=\left\langle x_{0} h+\lambda y_{0} e+\frac{1}{\lambda} z_{0} f\right\rangle .
$$




\section{Reduced lattice automorphisms}

Throughout this section, we suppose that $\phi$ is reduced with respect to $H,\left\{e_{\alpha} \mid \alpha \operatorname{root}\right\},<$.

LemMA 4.1. Let $\alpha$ be a root. Then there exists a root $\beta$ such that $(\alpha, \beta)=0$ and $\alpha+\beta$ is not a root.

Proof. We need only consider the case where $\alpha$ is a fundamental root at the end of a component of the Dynkin diagram since, for any $\alpha$, there exists an element of the Weyl group which transforms $\alpha$ into such a root. For such a root $\alpha$, there is a point $\beta$ of the Dynkin diagram not joined to $\alpha$ and the result follows.

LEMMA 4.2. For each root $\alpha$, there is $\lambda_{\alpha} \in F$ such that $\left\langle h_{\alpha}+x e_{\alpha}\right\rangle^{\varphi}=\left\langle h_{\alpha}\right.$ $+\lambda_{\alpha} x e_{\alpha}>$ for all $x \in F$, and $\lambda_{\alpha} \lambda_{-\alpha}=1$.

Proof. We choose a root $\beta$ such that $(\alpha, \beta)=0$ and $\alpha+\beta$ is not a root. There exist $k \in K_{\alpha} \cap K_{\beta}$ and $n \in K_{\alpha}$ such that $k$ and $n$ are linearly independent. Then $\left\langle k, n, e_{\alpha}\right\rangle$ is an abelian 3-dimensional subalgebra fixed under $\phi$. Therefore $\phi$ is semi-linear on $\left\langle k, n, e_{\alpha}\right\rangle$. But $\phi$ is linear on $\langle k, n\rangle$ and therefore also on the fixed subalgebra $\left\langle k, e_{\alpha}\right\rangle$. Since $\left\langle k, e_{\alpha}, e_{\beta}\right\rangle$ is also a 3 -dimensional abelian fixed subalgebra, it follows that $\phi$ is linear on $\left\langle e_{\alpha}, e_{\beta}\right\rangle$. Put $h=h_{\alpha}+h_{\beta}$. Every subspace of $\left\langle h, e_{\alpha}, e_{\beta}\right\rangle$ is a subalgebra and it follows that $\phi$ is also linear on $\left\langle h, e_{\alpha}, e_{\beta}\right\rangle$. Since $\phi$ fixes $\langle h\rangle,\left\langle e_{\alpha}\right\rangle$ and $\left\langle e_{\beta}\right\rangle, \phi$ is given on $\left\langle h, e_{\alpha}, e_{\beta}\right\rangle$ by

$$
\left\langle x h+y e_{\alpha}+z e_{\beta}\right\rangle^{\phi}=\left\langle x h+\lambda_{\alpha} y e_{\alpha}+\lambda_{\beta} z e_{\beta}\right\rangle
$$

for some $\lambda_{\alpha}, \lambda_{\beta} \in F$.

In the 3-dimensional projective geometry of subspaces of $\left\langle h_{\alpha}, h_{\beta}, e_{\alpha}, e_{\beta}\right\rangle$, $\left\langle h_{\alpha}, e_{\alpha}\right\rangle$ and $\left\langle h_{\beta}, e_{\beta}\right\rangle$ are skew lines. There is a unique transversal to them through $\left\langle h+x e_{\alpha}+y e_{\beta}\right\rangle$. This transversal $\left\langle h_{\alpha}+x e_{\alpha}, h_{\beta}+y e_{\beta}\right\rangle$ is a subalgebra since $(\alpha, \beta)=0$ and $\alpha+\beta$ is not a root. Since $\left\langle h_{\alpha}+x e_{\alpha}, h_{\beta}+y e_{\beta}\right\rangle^{\phi}$ is the transversal through $\left\langle h+x e_{\alpha}+y e_{\beta}\right\rangle^{\phi}=\left\langle h+\lambda_{\alpha} x e_{\alpha}+\lambda_{\beta} y e_{\beta}\right\rangle$,

$$
\begin{aligned}
\left\langle h_{\alpha}+x e_{\alpha}, h_{\beta}+y e_{\beta}\right\rangle^{n} & =\left\langle h_{\alpha}+\lambda_{\alpha} x e_{\alpha}, h_{\beta}+\lambda_{\beta} y e_{\beta}\right\rangle \text { and } \\
\left\langle h_{\alpha}+x e_{\alpha}\right\rangle^{\phi} & =\left\langle h_{\alpha}, e_{\alpha}\right\rangle \cap\left\langle h_{\alpha}+x e_{\alpha}, h_{\beta}+y e_{\beta}\right\rangle^{\phi} \\
& =\left\langle h_{\alpha}+\lambda_{\alpha} x e_{\alpha}\right\rangle .
\end{aligned}
$$

By Lemma 3.1, we have $\lambda_{\alpha} \lambda_{-\alpha}=1$.

COROLlaRY 4.3. The concept of a reduced lattice automorphism is independent of the choice of the root vectors $e_{\alpha}$.

ProOF. If $\phi$ is reduced and $\alpha$ is a fundamental root, then $\lambda_{\alpha}=1$ and $\phi$ is the identity on $\left\langle h_{\alpha}, e_{\alpha}\right\rangle$. For any choice $e_{\alpha}^{\prime}$ of the root vector, we have $\left\langle h_{\alpha}+e_{\alpha}^{\prime}\right\rangle^{\phi}$ $=\left\langle h_{\alpha}+e_{\alpha}^{\prime}\right\rangle$ as required. 
Lemma 4.4. For all $h \in H-K_{\alpha},\left\langle h+e_{\alpha}\right\rangle^{\phi}=\left\langle h+\lambda_{\alpha} e_{\alpha}\right\rangle$.

Proof. If $h \in H-K_{\alpha}$, then $h=u h_{\alpha}+k$ for some $k \in K_{\alpha}$ and $u \in F, u \neq 0$. By Lemma 4.2, the result holds for $k=0$. Suppose $k \neq 0$. Then

and

$$
\left\langle h+e_{\alpha}\right\rangle=\left\langle u h_{\alpha}+e_{\alpha}, k\right\rangle \cap\left\langle u h_{\alpha}+k, e_{\alpha}\right\rangle
$$

$$
\left\langle h+e_{\alpha}\right\rangle^{\phi}=\left\langle u h_{\alpha}+\lambda_{\alpha} e_{\alpha}, k\right\rangle \cap\left\langle u h_{\alpha}+k, e_{\alpha}\right\rangle=\left\langle h+\lambda_{\alpha} e_{\alpha}\right\rangle .
$$

LEMMA 4.5. Let $\alpha, \beta$ be independent roots and let $N_{\alpha, \beta}=\left\langle e_{\gamma}\right| \gamma=r \alpha+s \beta$, $r \geqq 0, s \geqq 0\rangle$. Then

$$
\left\langle x e_{\alpha}+y e_{\beta}, N_{\alpha, \beta}^{\prime}\right\rangle^{\phi}=\left\langle\lambda_{\alpha} x e_{\alpha}+\lambda_{\beta} y e_{\beta}, N_{\alpha, \beta}^{\prime}\right\rangle
$$

for all $x, y \in F$.

ProOF. Put

$$
p=(\alpha, \alpha) \frac{(\beta, \beta)-(\alpha, \beta)}{(\alpha, \alpha)(\beta, \beta)-(\alpha, \beta)^{2}}, \quad q=(\beta, \beta) \frac{(\alpha, \alpha)-(\alpha, \beta)}{(\alpha, \alpha)(\beta, \beta)-(\alpha, \beta)^{2}}
$$

and $h=p h_{\alpha}+q h_{\beta}$. Then $h e_{\alpha}=e_{\alpha}$ and $h e_{\beta}=e_{\beta}$. Put $N=N_{\alpha, \beta}$ and $U=\langle h, N\rangle$. Then $N$ is a fixed nilpotent subalgebra. Since $N^{\prime}$ is normalised by $H, N^{\prime}$ is a sum of root spaces and is therefore fixed. But $N^{\prime}$ is an ideal of $U$ and every subspace of $U / N^{\prime}$ is a subalgebra. Hence $\phi$ is semi-linear on $U / N^{\prime}$. By Lemma 4.4, we have

$$
\left\langle z h+x e_{\alpha}\right\rangle^{\phi}=\left\langle z h+\lambda_{\alpha} x e_{\alpha}\right\rangle,\left\langle z h+y e_{\beta}\right\rangle^{\phi}=\left\langle z h+\lambda_{\beta} y e_{\beta}\right\rangle
$$

and it follows that

$$
\left\langle z h+x e_{\alpha}+y e_{\beta}, N^{\prime}\right\rangle^{\phi}=\left\langle z h+\lambda_{\alpha} x e_{\alpha}+\lambda_{\beta} y e_{\beta}, N^{\prime}\right\rangle
$$

for all $x, y, z \in F$.

Lemma 4.6. There exists $t=t(H) \in F, t \neq 0$, independent of $\alpha$, such that $\left\langle k+e_{\alpha}\right\rangle^{\phi}=\left\langle k+t \lambda_{\alpha} e_{\alpha}\right\rangle$ for all $k \in K_{\alpha}$.

ProOF. Since $\left\langle K_{\alpha}, e_{\alpha}\right\rangle$ is a fixed abelian subalgebra of dimension $\geqq 3, \phi$ is semi-linear on $\left\langle K_{\alpha}, e_{\alpha}\right\rangle$. But $\phi$ is the identity on $K_{\alpha}$ and fixes $\left\langle e_{\alpha}\right\rangle$. Hence $\phi$ is given on $\left\langle K_{\alpha}, e_{\alpha}\right\rangle$ by $\left\langle k+e_{\alpha}\right\rangle^{\phi}=\left\langle k+\mu_{\alpha} e_{\alpha}\right\rangle$ for all $k \in K_{\alpha}$ and some $\mu_{\alpha} \in F, \mu_{\alpha} \neq 0$ independent of $k$. We have to prove that $\mu_{\alpha} / \lambda_{\alpha}$ is independent of $\alpha$.

Let $\alpha, \beta$ be independent roots. Take $k \in K_{\alpha} \cap K_{\beta}, k \neq 0$, and put $V=\langle k, N\rangle$ where $N=N_{\alpha, \beta}$. Then $N^{\prime}$ is an ideal of $V, V / N^{\prime}$ is abelian, and it follows that $\phi$ is given on $V / N^{\prime}$ by

$$
\left\langle z k+x e_{\alpha}+y e_{\beta}, N^{\prime}\right\rangle^{\phi}=\left\langle z k+\mu_{\alpha} x e_{\alpha}+\mu_{\beta} y e_{\beta}, N^{\prime}\right\rangle .
$$

Comparing this with the description of $\phi$ on $\left\langle e_{\alpha}, e_{\beta}, N^{\prime}\right\rangle$ given by Lemma 4.5 , we get $\mu_{\alpha} / \lambda_{\alpha}=\mu_{\beta} / \lambda_{\beta}$. 
LEMMA 4.7. Let $\sigma=\exp \left(\theta \operatorname{ad} e_{\beta}\right)$ where $\theta \in F$. Suppose $\lambda_{\beta}=1$. Then $\phi$ is reduced with respect to $H^{\sigma}$ and $<^{\sigma}, \lambda_{\alpha^{\sigma}}=\lambda_{\alpha}$ for all roots $\alpha$ of $H$, and $t\left(H^{\sigma}\right)=t(H)$.

PROOF. $H=\left\langle h_{\beta}, K_{\beta}\right\rangle$ and $H^{\sigma}=\left\langle h_{\beta}+\theta e_{\beta}, K_{\beta}\right\rangle$. We have that $\phi$ is the identity on $K_{\beta}$ and, by Lemma $4.4,\left\langle h_{\beta}+k+\theta e_{\beta}\right\rangle^{\phi}=\left\langle h_{\beta}+k+\lambda_{\beta} \theta e_{\beta}\right\rangle$ for all $k \in K_{\beta}$. Since $\lambda_{\beta}=1, \phi$ is the identity on $H^{\sigma}$. It follows that, for every root $\gamma$ of $H^{\sigma}$, either $\left\langle e_{\gamma}\right\rangle^{\phi}=\left\langle e_{\gamma}\right\rangle$ or $\left\langle e_{\gamma}\right\rangle^{\phi}=\left\langle e_{-\gamma}\right\rangle$. But $\gamma=\alpha^{\sigma}$ for some root $\alpha$ of $H$ and $e_{\gamma}=e_{\alpha}^{\sigma} \in N_{\alpha, \beta}$. Since $e_{\alpha}^{\sigma} \notin N_{-\alpha, \beta},\left\langle e_{\gamma}\right\rangle^{\phi} \neq\left\langle e_{-\gamma}\right\rangle$ and we have $\left\langle e_{\gamma}\right\rangle^{\phi}=\left\langle e_{\gamma}\right\rangle$ for all roots $\gamma$ of $H^{\sigma}$.

By Lemma 4.4, for some $\lambda_{\gamma} \in F$, we have $\left\langle h+e_{\gamma}\right\rangle^{\phi}=\left\langle h+\lambda_{\gamma} e_{\gamma}\right\rangle$ for all $h \in H^{\sigma}-K_{\gamma}$. (The proof of Lemma 4.4 makes no use of the assumption that $\lambda_{\alpha}=1$ for all fundamental roots $\alpha$.) Trivially, $\lambda_{\beta \sigma}=\lambda_{\beta}$.

For $\alpha \neq \pm \beta$, take $h \in K_{\beta}, h \notin K_{\alpha^{*}}$. Then $h \in H^{\sigma}-K_{\alpha^{\sigma}}$ and

$$
\begin{aligned}
\left\langle h+\lambda_{\alpha} \sigma e_{\alpha}^{\sigma}, N_{\alpha, \beta}^{\prime}\right\rangle & =\left\langle h+e_{\alpha}^{\sigma}, N_{\alpha, \beta}^{\prime}\right\rangle^{\phi}=\left\langle h+e_{\alpha}, N_{\alpha, \beta}^{\prime}\right\rangle^{\phi} \\
& =\left\langle h+\lambda_{\alpha} e_{\alpha}, N_{\alpha, \beta}^{\prime}\right\rangle .
\end{aligned}
$$

Therefore $\lambda_{\alpha^{\sigma}}=\lambda_{\alpha^{\circ}}$. In particular, if $\alpha^{\sigma}$ is fundamental with respect to $<^{\sigma}$, then $\lambda_{\alpha^{\sigma}}=1$. Thus $\phi$ is reduced with respect to $H^{\sigma}$ and $<^{\sigma}$.

Take $k \in K_{\beta^{\sigma}}=K_{\beta}$. Since $e_{\beta^{\sigma}}=e_{\beta}$, we have

$$
\left\langle k+e_{\beta^{\sigma}}\right\rangle^{\phi}=\left\langle k+e_{\beta}\right\rangle^{\phi}=\left\langle k+t \lambda_{\beta} e_{\beta}\right\rangle=\left\langle k+t \lambda_{\beta \sigma} e_{\beta \sigma}\right\rangle
$$

and $t\left(H^{\sigma}\right)=t(H)$.

LEMMA 4.8. For all $\alpha, \lambda_{\alpha}=1$. The concept of a reduced lattice automorphism is independent of the ordering of the roots.

Proof. It is sufficient to show that $\lambda_{\alpha}=\lambda_{\beta}=1$ implies $\lambda_{\alpha+\beta}=1$. Since we may suppose $\alpha+\beta$ is a root (otherwise we have nothing to prove), we have $e_{\alpha} e_{\beta}=v e_{\alpha+\beta}$ and $v \neq 0$. Put $\sigma=\exp \left(1 / v \operatorname{ad} e_{\beta}\right)$ and $N=N_{\alpha, \beta}$. Since the term $\Gamma^{3} N$ of the descending central series of $N$ is normalised by $H$, it is a sum of root spaces and is therefore fixed. Since $\lambda_{\alpha}=1$ and $N_{\alpha, \alpha+\beta}^{\prime} \subseteq \Gamma^{3} N$, we have by Lemma 4.5 that $\left\langle e_{\alpha}+e_{\alpha+\beta}, \Gamma^{3} N\right\rangle^{\phi}=\left\langle e_{\alpha}+\lambda_{\alpha+\beta} e_{\alpha+\beta}, \Gamma^{3} N\right\rangle$. But

$$
\begin{aligned}
\left\langle e_{\alpha}+e_{\alpha+\beta}, \Gamma^{3} N\right\rangle^{\phi} & =\left\langle e_{\alpha}^{\sigma}, \Gamma^{3} N\right\rangle^{\phi}=\left\langle e_{\alpha}^{\sigma}, \Gamma^{3} N\right\rangle \\
& =\left\langle e_{\alpha}+e_{\alpha+\beta}, \Gamma^{3} N\right\rangle .
\end{aligned}
$$

Since $e_{\alpha+\beta} \notin \Gamma^{3} N$, it follows that $\lambda_{\alpha+\beta}=1$.

LEMMA 4.9. Let $H, H^{*}$ be Cartan subalgebras of $L$, and suppose $\phi$ is reduced with respect to $H$. Then $\phi$ is reduced with respect to $H^{*}$ and $t(H)=t\left(H^{*}\right)$. The concept of a reduced lattice automorphism is independent of the choice of Cartan subalgebra.

ProOF. By Lemmas 4.7, 4.8, it is sufficient to prove the existence of a chain of Cartan subalgebras $H_{0}=H, H_{1}, \cdots, H_{n}=H^{*}$ and root vectors $e_{\alpha}^{i}$ of $H_{i}(i=0,1, \cdots$, 
$n-1)$ such that $H_{i+1}=H_{i} \exp \left(\lambda_{i}\right.$ ad $\left.e_{\alpha}^{i}\right)$. By Jaco'son [3] p. 288 Exercise 18, there exist roots $\alpha_{1}, \cdots, \alpha_{n}$ of $H$, not necessarily distinct, and $\lambda_{i} \in F$ such that

Put

$$
H^{*}=\exp \left(\lambda_{1} \text { ad } e_{\alpha_{1}}\right) \exp \left(\lambda_{2} \text { ad } e_{\alpha_{2}}\right) \cdots \exp \left(\lambda_{n} \operatorname{ad} e_{\alpha_{n}}\right) \text {. }
$$

$$
\sigma_{i}=\exp \left(\lambda_{n-i} \text { ad } e_{\alpha_{-i n}}\right) \exp \left(\lambda_{n-i+1} \operatorname{ad} e_{\alpha_{n-i+1}}\right) \cdots \exp \left(\lambda_{n} \operatorname{ad} e_{\alpha_{n}}\right),
$$

$H_{i+1}=H^{\sigma_{i}}$ and $e_{\alpha}^{i+1}=e_{\alpha_{n-i-1}}^{\sigma_{i}}$. Then ad $e_{\alpha}^{i+1}=\sigma_{i}^{-1}\left(\right.$ ad $\left.e_{\alpha_{n-i-1}}\right) \sigma_{\mathrm{l}}$ and $\sigma_{i} \exp$ $\left(\lambda_{i+1}\right.$ ad $\left.e_{\alpha}^{i+1}\right)=\sigma_{i+1}$. Thus we have $e_{\alpha}^{i}$ a root vector for $H_{i}, H_{i+1}=H_{i} \exp \left(\lambda_{i}\right.$ ad $\left.e_{\alpha}^{i}\right)$ and $H_{n}=H^{*}$.

LEMMA 4.10. Suppose $\phi$ is a reduced lattice automorphism of L. Let $t=t(H)$ for some Cartan subalgebra $H$ of $L$. Then $\langle x\rangle^{\phi}=\left\langle x_{s}+t x_{n}\right\rangle$ for all $x \in L$.

Proof. (a) Suppose $x$ is semi-simple. Then there exists a Cartan subalgebra $H$ of $L$ such that $x \in H$. By Lemma $4.9, \phi$ is reduced with respect to $H$ and therefore $\langle x\rangle^{\phi}=\langle x\rangle$.

(b) Suppose $x$ is nil. Then there exist $h, y \in L$ such that $h x=x, h y=-y$ and $x y=h$. All the elements $h+\lambda x$ are semi-simple. Therefore all the subalgebras $\langle h+\lambda x\rangle$ are fixed, and it follows that $\langle x\rangle$ is fixed.

(c) Suppose $x_{s}$ and $x_{n}$ are non-zero. Take a Cartan subalgebra $H$ such that $x_{s} \in H$. Then $x_{n}=\Sigma_{\alpha} \mu_{\alpha} e_{\alpha}$ where $\mu_{\sigma} \in F$ and $e_{\alpha}$ is a root vector for the root $\alpha$ of $H$. For some ordering of the roots, the roots $\alpha$ for which $\mu_{\alpha} \neq 0$ are all positive. Since $x_{s} x_{n}=0$, if $\mu_{\alpha} \neq 0$, then $\alpha\left(x_{s}\right)=0$. Let $\gamma$ be the largest root for which $\gamma\left(x_{s}\right)=0$. Then $\left\langle x_{s}+\lambda e_{\gamma}\right\rangle^{\phi}=\left\langle x_{s}+t \lambda e_{\gamma}\right\rangle$ by Lemma 4.6. Thus the result holds if $x_{n}=\lambda e_{\gamma}$ for some $\lambda \in F$. If $x_{n}$ and $e_{\gamma}$ are linearly independent, then $\left\langle x_{s}, x_{n}, e_{\gamma}\right\rangle$ is a fixed 3-d.mensional abelian subalgebra. Every element of $\left\langle x_{n}, e_{y}\right\rangle$ is nil. Thus $\phi$ is the identity on $\left\langle x_{n}, e_{\gamma}\right\rangle$. From $\phi$ linear on $\left\langle x_{s}, x_{n}, e_{\gamma}\right\rangle$ and $\left\langle x_{s}+e_{\gamma}\right\rangle^{\phi}=\left\langle x_{s}+t e_{\gamma}\right\rangle$, it now follows that $\left\langle x_{s}+x_{n}\right\rangle^{\phi}=\left\langle x_{s}+t x_{n}\right\rangle$.

This completes the proof of the direct part of the theorem.

\section{The converse}

In this section, we suppose that $L$ is a semi-simple Lie algebra over a (not necessarily algebraically closed) field of characteristic 0 . We take some $t \in F, t \neq 0$ and put $\tau=\tau(t)$ and we prove that $\tau$ induces a reduced lattice automorphism of $L$.

LEMMA 5.1. Let $A=\langle\langle a, b\rangle\rangle$ be the subalgebra of $L$ generated $b y a, b$. Then

(a) $a_{s} b_{s}, a_{s} b_{n}, a_{n} b_{s}, a_{n} b_{n} \in A^{\prime}$.

(b) There exists $u \in A^{\prime}$ such that $a_{s}(b+u)=0$.

(c) If $A$ is soluble, there exist $c, d \in A$ such that $c-a, d-b \in A^{\prime}$ and $c_{s} d=c d_{s}=0$. 
Proof. (a) For all polynomia's $f(x)$ without constant term, $b f(\operatorname{ad} a) \in A^{\prime}$. In particular, $b$ ad $a_{s} \in A^{\prime}$ and $b$ ad $a_{n} \in A^{\prime}$. Thus $a_{s} b, a_{n} b \in A^{\prime}$. Therefore $a_{s} g(\operatorname{ad} b) \in A^{\prime}$ $a_{n} g(\operatorname{ad} b) \in A^{\prime}$ for all polynomials $g(x)$ without constant term. Therefore $a_{s} b_{s}$, $a_{s} b_{n}, a_{n} b_{s}, a_{n} b_{n} \in A^{\prime}$.

(b) Let $m(x)=x f(x)$ be the minimum polynomial of ad $a_{s}$. Then $x$ does not divide $f(x)$, and there exist polynomials $p(x), q(x)$ such that $x p(x)+f(x) q(x)=1$. Put $u=-b$ ad $a_{s} p\left(\operatorname{ad} a_{s}\right)$. Then $u \in A^{\prime}, b+u=b f\left(\operatorname{ad} a_{s}\right) q\left(\operatorname{ad} a_{s}\right)$ and $a_{s}(b+u)$ $=0$.

(c) By (b), there exist $u \in A^{\prime}$ such that $(a+u) b_{s}=0$. Put $c=a+u$. Since ad $b_{s}$ commutes with ad $c$, it commutes with all polynomials in ad $c$, in particular with ad $c_{s}$. By the argument of (b), there exists a polynomial $f(x)$ such that, for $v=b_{n} f\left(\operatorname{ad} c_{s}\right)$, we have $c_{s}\left(b_{n}+v\right)=0$. Clearly $v \in A^{\prime}$. Let $K$ be the algebraic closure of $F$ and let $L_{K}$ be the algebra obtained from $L$ by extension of the field. Since $A$ is soluble, $A$ is contained in a Borel subalgebra $B$ of $L_{K} . B^{\prime}$ is precisely the set of all nil elements in $B$. It follows that every element of $A^{\prime}$ is nil and that the sum of two nil elements of $A$ is again nil. Thus $b_{n}+v$ is nil. Since ad $b_{s}$ commutes with ad $c_{s}, v b_{s}=b_{n} b_{s} f\left(\operatorname{ad} c_{s}\right)=0$. Thus $b_{s}\left(b_{n}+v\right)=0$ and $b_{s}$ and $b_{n}+v$ are the semi-simple and nil parts respectively of $d=b+v$.

LEMma 5.2. Let $U$ be a subset of $L, P=\langle\langle U\rangle\rangle$ and $Q=\left\langle\left\langle U^{x}\right\rangle\right\rangle$. Then $P^{\prime}=Q^{\prime}$.

Proof. For all $u, v \in U$, we have

$$
u^{\tau} v^{\tau}=\left(u_{s}+t u_{n}\right)\left(v_{s}+t v_{n}\right)=u_{s} v_{s}+t\left(u_{n} v_{s}+u_{s} v_{n}\right)+t^{2} u_{n} v_{n}
$$

and by Lemma 5.1(a), $u^{\tau} v^{\tau} \in P^{\prime}$. For all $u \in U$ and $a \in P^{\prime}$, we have $u^{\tau} a=u_{s} a+$ $t u_{n} a \in P^{\prime}$ by Lemma 5.1 (a). It follows that $Q^{\prime} \subseteq P^{\prime}$. Applying this to $\tau^{-1}=\tau(1 / t)$, we get $P^{\prime} \subseteq Q^{\prime}$.

Lemma 5.3. Let $A$ be a soluble subalgebra of L. Then $A^{\tau}$ is a subalgebra of $L$ and $\operatorname{dim} A^{\mathfrak{t}}=\operatorname{dim} A$.

Proor. Since all elements of $A^{\prime}$ are nil, $A^{\prime \tau}=A^{\prime} . A^{\tau}$ is closed with respect to multiplication since, by Lemma $5.2,\left\langle\left\langle A^{\tau}\right\rangle\right\rangle^{\prime}=A^{\prime}$. We have to prove that $A^{\tau}$ is a subspace of the same dimension as $A$.

(1) We use induction on $\operatorname{dim} A$ to prove that $(a+b)^{\tau}-a^{\tau}-b^{\tau} \in A^{\prime}$ for all $a, b \in A$. The assertion is trivial if $\operatorname{dim} A=1$. We can work in $\langle\langle a, b\rangle\rangle$, so we may suppose $A=\langle\langle a, b\rangle\rangle$.

CASE 1. Suppose $b \in A^{\prime}$. If $b=0$, the result is trivial, so we suppose $b \neq 0$. Since $A^{\prime} \neq 0$, we have $\left.A^{\prime}\right\rangle A^{\prime \prime}$. As $A=\left\langle a, A^{\prime}\right\rangle$ and $b \in A^{\prime}=a A^{\prime}+A^{\prime \prime}$, we have $b=a u+v$ for some $u \in A^{\prime}, v \in A^{\prime \prime}$. Put

$$
c=a \exp (\operatorname{ad} u)=a+a u+\frac{1}{2}(a u) u+\cdots .
$$


Then $c=a+b+w$ where $w \in A^{\prime \prime}$. We have

and

$$
\begin{aligned}
& c_{s}=a_{s} \exp (\operatorname{ad} u) \equiv a_{s} \bmod A^{\prime} \\
& c_{n}=a_{n} \exp (\operatorname{ad} u) \equiv a_{n} \bmod A^{\prime},
\end{aligned}
$$

$$
c^{\tau}-a^{\tau}=\left(c_{s}-a_{s}\right)+t\left(c_{n}-a_{n}\right) \equiv 0 \bmod A^{\prime} .
$$

Since $w \in A^{\prime \prime}\left\langle A^{\prime},\langle\langle c, w\rangle\rangle\left\langle A\right.\right.$ and by induction, $(c-w)^{\tau}-c^{\tau}+w^{\tau} \in\langle\langle c, w\rangle\rangle^{\prime}$. But $b^{\tau}, w^{\tau} \in A^{\prime}$ and therefore

$$
(a+b)^{\tau}-a^{\tau}-b^{\tau}=(c-w)^{\tau}-a^{\tau}-b^{\tau} \equiv c^{\tau}-a^{\tau} \equiv 0 \bmod A^{\prime} .
$$

CASE 2. We now consider general $a, b$. By Lemma 5.1(c), there exist $c, d \in A$ such that $c-a, d-b \in A^{\prime}$ and $c_{s} d=c d_{s}=0$. Put $u=c-a, v=d-b$. Since $c_{s} d_{s}=0, c_{s}+d_{s}$ is semi-simple. Since $c_{n}, d_{n}$ are nil and $A$ is soluble, $c_{n}+d_{n}$ is nil. But $\left(c_{s}+d_{s}\right)\left(c_{n}+d_{n}\right)=0$. Therefore $\left(c_{s}+d_{s}\right)$ and $\left(c_{n}+d_{n}\right)$ are the semi-simple and nil parts of $c+d$, and we have $(c+d)^{\tau}-c^{\tau}-d^{\tau}=0$. Trivially, $(u+v)^{\tau}$ $=u^{\tau}+v^{\tau}$ since $u, v, u+v$ are nil. By Case 1 , we have

$$
\begin{aligned}
((c+d)-(u+v))^{\mathfrak{\tau}} & \equiv(c+d)^{\tau}-(u+v)^{\tau} \\
a^{\tau} & =(c-u)^{\tau} \equiv c^{\tau}-u^{\tau} \\
b^{\tau} & =(d-v)^{\tau} \equiv d^{\tau}-v^{\tau} \bmod A^{\prime} .
\end{aligned}
$$

Thus

$$
\begin{aligned}
(a+b)^{\tau}-a^{\tau}-b^{\tau} & =((c+d)-(u+v))^{\tau}-a^{\tau}-b^{\tau} \\
& \equiv(c+d)^{\tau}-(u+v)^{\tau}-c^{\tau}+u^{\tau}-d^{\tau}+v^{\tau} \\
& \equiv 0 \bmod A^{\prime}
\end{aligned}
$$

(2) For any $a \in A$, by (1) we have $\left(a+A^{\prime}\right)^{\tau} \subseteq a^{\tau}+A^{\prime}$. Applying this to $\tau^{-1}$ and the soluble subalgebra $\left\langle a^{\tau}, A^{\prime}\right\rangle$, we get $\left(a+A^{\prime}\right)^{\tau}=a^{\tau}+A^{\prime}$. Put $B=\left\langle A^{\tau}\right\rangle$. Then $\tau$ induces a map $\tau_{1}: A \mid A^{\prime} \rightarrow B / A^{\prime}$. By (1), $\tau_{1}$ is linear and im $\tau_{1}$ is a subspace of $B / A^{\prime}$. But $\left\langle a^{\tau}+A^{\prime} \mid a \in A\right\rangle=B / A^{\prime}$. Thus im $\tau_{1}=B / A^{\prime}$ and it follows that $A^{\tau}=B$. Since $\tau_{1}$ is invertible, $\operatorname{dim} B / A^{\prime}=\operatorname{dim} A / A^{\prime}$ and the result follows.

LeMmA 5.4. Let $A$ be any subalgebra of $L$. Then $A^{\tau}$ is a subalgebra of $L$ and $\operatorname{dim} A^{\tau}=\operatorname{dim} A$.

Proof. Let $R$ be the radical of $A$ and let $S$ be a Levi factor. Then $A=R \oplus S$ as vector space. By Lemma 5.3, $R^{\tau}$ is a subalgebra of $L$ and $R^{\tau}$ is soluble since $R^{\tau \prime}=R^{\prime}$. Since $S$ is semi-simple, we have $S=S^{\prime}=\left\langle\left\langle S^{\tau}\right\rangle\right\rangle^{\prime}$ and so $S^{\tau} \subseteq S$. Applying this to $\tau^{-\tau}$, we get $S^{\tau-1} \subseteq S$ and so $S^{\tau}=S$.

For any $s \in S,\langle s, R\rangle$ is a soluble subalgebra and $\langle s, R\rangle^{\prime} \subseteq R$. By Lemma 5.3 $\langle s, R\rangle^{\tau}$ is a subalgebra and 


$$
\langle s, R\rangle^{\tau \prime}=\langle s, R\rangle^{\prime}=\langle s, R\rangle^{\prime \tau} \subseteq R^{\tau} .
$$

Thus $S^{\tau} R^{\tau} \subseteq R^{\tau}$ and $R^{\tau}+S$ is a subalgebra. Sinze $R^{\tau}$ is a soluble ideal of $R^{\tau}+S$ $R^{\tau} \cap S=0$ and $\operatorname{dim}\left(R^{\tau}+S\right)=\operatorname{dim}(R+S)$.

Suppose $a \in A$. Then $a=r+s$ for some $r \in R, s \in S$, and $a \in\langle s, R\rangle$. Hence $a^{\tau} \in\langle s, R\rangle^{\tau} \subseteq R^{\tau}+S$ since $\langle s, R\rangle^{\tau}$ is a subalgebra. Thus $(R+S)^{\tau} \subseteq R^{\tau}+S$. Applying this to $\tau^{-1}$ gives the reverse inequality. Therefore $A^{\tau}=R^{\tau}+S$ and so is a subalgebra.

This completes the proof that $\tau$ induces a reduced lattice automorphism of $L$, and so completes the proof of the theorem.

\section{References}

[1] D. W. Barnes, 'Lattice isomorphisms of Lie algebras', J. Austral. Math. Soc. 4 (1964), 470-475.

[2] D. W. Barnes and G. E. Wall, 'On normaliser preserving lattice isomorphisms between nilpotent groups', J. Austral. Math. Soc. 4 (1964), 454-469.

[3] N. Jacobson, Lie Algebras (Interscience Tracts No. 10, 1962).

Department of Pure Mathematics

University of Sydney

A ustralia 